\title{
IoT-based Monitoring System using Tri-level Context Making for Smart Home Services
}

\author{
Mubeena Begum ${ }^{1}$, Sagara T V ${ }^{2}$, Fehmida Begum ${ }^{3}$ \\ ${ }^{I}$ (Pg Student, Dept Of Ece, Bitm College, Bellary, (Vtu Belgaum), Karnataka, India) \\ ${ }^{2}$ (Assistant Professor, Dept Of Ece, Bitm College, Bellary, (Vtu Belgaum), Karnataka, India) \\ ${ }^{3}$ (Student, Dept Of Ece, Pdit college Hospet, , (Vtu Belgaum), Karnataka, India)
}

\begin{abstract}
The advancements in Wireless Sensor Networks (WSN) \& Internet technologies, a new trend in era of ubiquity is being realized. The Enormous increase in users of Internet \& modifications on internetworking technologies enable networking of everyday objects. The "Internet of Things (IoT)" is about physical items talking each other, machine to machine communications and person to computer communications will extended to "things". The Key technologies that drive future IoT related to Smart sensor technologies including Nanotechnology, WSN and Miniaturization. The integrated network architecture and the interconnecting mechanisms for reliable measurements of parameters by smart sensors and transmission of data via internet .The system which can use to update the values or parameters in home by internet via different communication protocols but in our proposed system we are using Ethernet protocol which is fastest protocol currently available in market. In our system we have designed two sections one has AT89S52 microcontroller interfaced with sensors. The other section has ARM7 microcontroller connected with Ethernet protocol. The two sections can communicate by Zigbee wireless module in which Sensor Node sends data to Ethernet section. The Ethernet section will update data in to internet via Ethernet protocol. So we can view status of parameters and can control it via internet.
\end{abstract}

Keywords: ARM7 microcontroller, AT89S52 microcontroller, Internet of things, WSN, zigbee.

\section{Introduction}

As advanced wired and wireless network technologies have developed, internet-connected mobile devices such as smart phones and tablets are now in general use. As a result, a new concept, Internet of Things (IoT), was introduced and it has received attention over the past few years. In general, IoT is an information sharing environment where objects in every-day life are connected to wired and wireless networks. Recently, it is used not only in the field of consumer electronics and appliances but also in other various fields such as a healthcare, smart home, smart car, energy system, and industrial security.

The IoT used to be compared to machine to machine (M2M) technology. According to the IoT global standard initiative (IoT-GSL), in the International Telecommunications Union (ITU-T), the IoT is not limited to physical objects but includes virtual objects such as contents. This characteristic of IoT is a typical difference when compared with M2M. In short, the M2M revolves around a machine which is the main object, but the IoT is introduced around an environment that has things or object surrounding people. According to [1], IoT means that each object or thing is connected and communicates through the Internet without human intervention, and the ultimate goal of IoT is to enhance the quality of human life. In human life, fields that are applied with the IoT technology are homes and buildings, which are defined as smart homes.

In past home automations, a network topology consisted of sensor nodes for data collection and transmission and servers or gateways for information collection and analysis [2], and the relationship between a sensor and an actuator was clearly defined [3]. On the other hand, in the IoT environment, a sensor and an actuator are not as clearly separated but rather defined as an individual object or thing. Thus, each object or thing is a sensor but also plays the role of the actuator, which should collect information while at the same time providing information to objects such as user devices, cloud servers, and other IoT. Such difference is one of the most definitive features that distinguish the IoT-based smart homes and the past home automations. Therefore, in the future smart homes, a new type of IoT is needed to provide various services and to improve human life, without user intervention. This paper proposes an IoT-based monitoring system using tri-level context making model for smart home services. It also demonstrates a context-based smart home service using an actual prototype and service scenario for future IoT environments. The main purpose of this paper is to demonstrate future IoT technologies and propose direction of smart home services. 


\section{Iot-Based Monitoring System}

From such perspective of the introduction, the IMS was designed to collect and analyze data, share information and create context in an IoT environment. Fig. 1 shows the hardware and software architecture of IMS and the difference between the home automation and the IoT-based smart home network topology. The IMS consists of the sensor part that measures environmental information, the communication part for connectivity, and the main process part that processes data and context. The sensor part is equipped with five sensors measuring temperature, humidity, gas, luminance, and movement. The communication part supports Wi-Fi and Bluetooth to interact with mobile devices, cloud servers, and other objects. Moreover, the IMS can provide collected and analyzed information via a webpage because it can be operated as a web server. The main processor part, equipped with the tri-level context making model, analyzes the collected data, creates context, and informs users by making a beeping sound and with an LED light according to context-aware results.

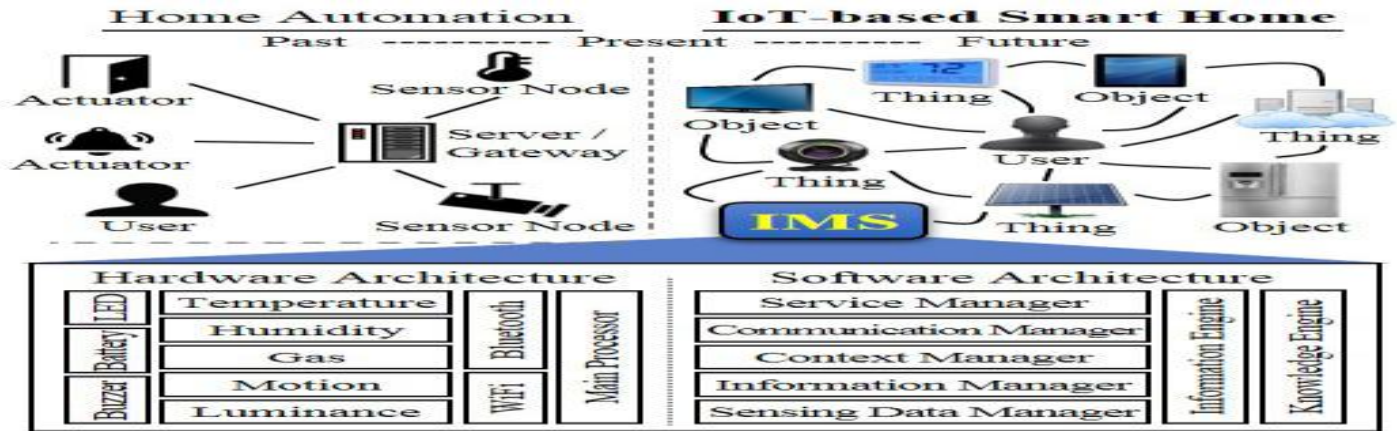

Fig.1. Architecture of IMS and the difference between home automation and IoT-based smart home network topology.

\section{Implementation}

The proposed IMS using the tri-level context making model was designed with open source hardware and software in order to expand into future IoT. Two different scenarios were tested as IMS-based smart home services. The first was a disaster management service. In this scenario, if specific situations such as fires, gas leakage, and break-ins occurred, users can be aware of them through the alarming of LED lights, beep buzzers, and remote notification. The second scenario was a smart home health care service such as deep sleep management and comfortable house management. This service analyzes information necessary for the maintenance of pleasant living environments and health promotions, and then provides services that require certain actions such as ventilation, temperature control, and indoor exercise in the same way as the alarming service of the first scenario. Fig. 2 presents the prototype of IMS and the graphical user interface of a mobile application. Through the mobile application, users can realize what type of actions are required to achieve the Purpose of a service.
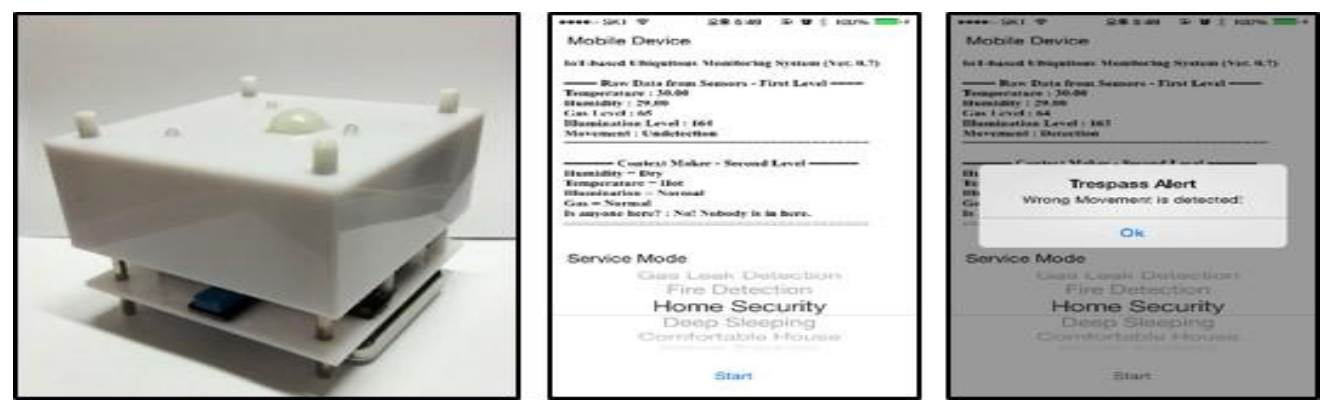

Fig.2. Prototype of IoT-based monitoring system

\section{Tri-Level Context Making Model}

The tri-level context making model plays a role of generating context from collected data depending on the type and purpose of service domains. For guaranteeing quality of service $(\mathrm{QoS})$ in various service domains, the proposed context making model is service oriented and divided into three levels. Each level of the proposed context making model has different service types, which are a simple monitoring service, an automatic control service, and a user-centric service. Fig. 3 shows the concept of the proposed context making model. The first step is the data acquisition in which data measured from the sensor is collected and calibrated. The second step is the information processing. In this step, the information engine provides appropriate threshold values according to sensor characteristics, and the collected data is processed into information which is low-level 
context. The last step is the context making. The aim of this step is to generate the situation-aware or context aware information based on the knowledge engine. This engine transforms the information into high-level contexts depending on the service type. And in this step, the IMS requires an appropriate user action according to each generated context.

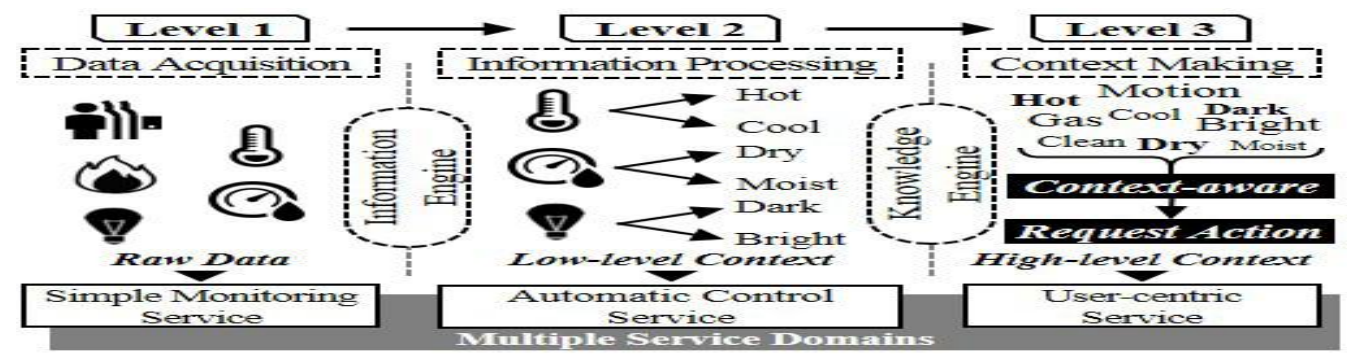

Fig.3. Concept of the Tri-level Context Making Model

As an example of the context making process, if a gas data and a temperature data are collected in the first step, the proposed context model determines whether the air quality is fresh, moderate or poor according to the threshold value in the second step. The low-level context related to the temperature is also determined to be high, low, or normal. In the last step, the high-level context is generated based on the knowledge engine to see whether it is a fire situation where gas is detected and temperature is rising, or a ventilation-requiring situation due to polluted air. The knowledge engine decides the most appropriate situation based on context-aware information that is initially learned and differs depending on characteristics of the service domains.

\section{Proposed System:}

In this system we have designed four different sections three has 8 bit microcontrollers interfaced with sensors. The other section has Arm microcontroller connected with the internet protocol through PC. All the sections can communicate with Zigbee wireless module, in which the Sensor Nodes sends the data to the internet section.The internet section will update the data in to the internet via internet protocol. So we can view status of the parameter via internet and here we are also propose a actuator mechanism to control the abnormal parameter, and those actuators will be operated via internet protocol.

Sensor Section-1:

\section{Block Diagram}

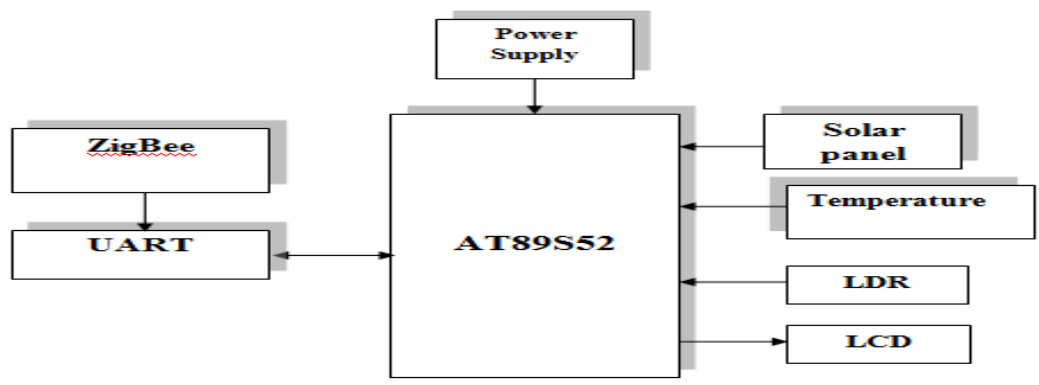

Sensor Section-2:

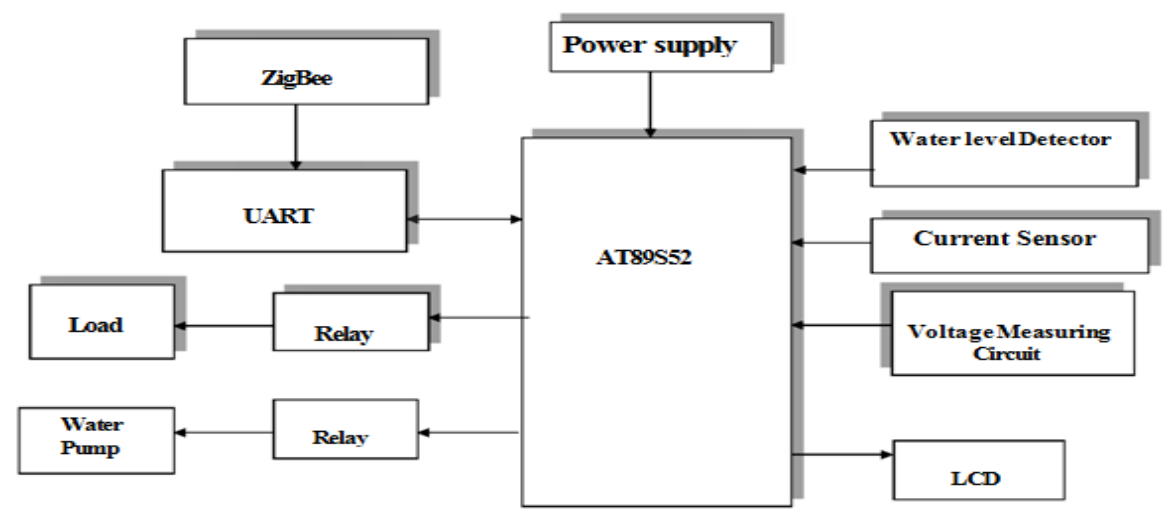




\section{Sensor Section-3:}

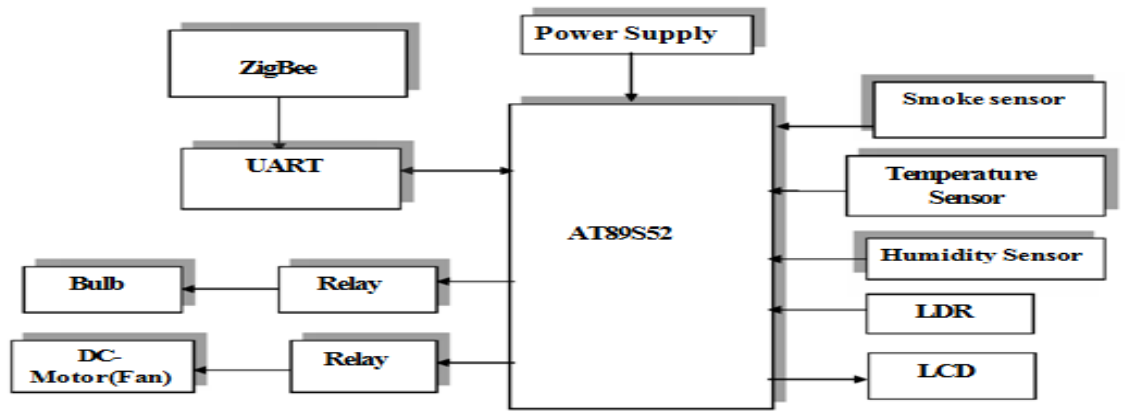

\section{Co-ordinator/ internet Section:}

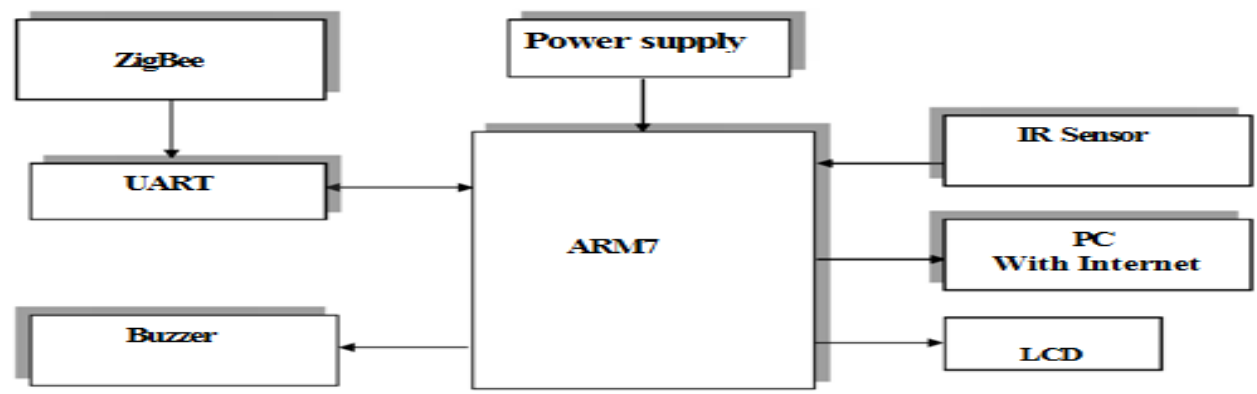

\section{Conclusion}

In order to enrich daily lives with the IoT, a necessary technology is one that collects and analyzes much different information around us through various sensors. Moreover, creating and managing context, which is information with significance, will be the core technology to develop future IoT-based smart home services. In this project, the IMS using a tri-level context making model for future smart home services was proposed. In the future IoT environment, collecting data and generating information from sensors are important because new services and various business models will appear. In order for the IoT to be fused into life and move forward, new IoT service designs and context analysis are also important. Therefore, the next step for this study will be reinforcing the knowledge engine, processing data from additional sensors, and visualizing data and information.

\section{Reference}

[1]. C. Perera, A. Zaslavsky, P. Christen, and D. Georgakopoulos, "Context Aware Computing for the Internet of Things: A Survey, "Communications Surveys \& Tutorials, IEEE, vol.16, no.1, pp.414-454, First Quarter 2014

[2]. K. Lee and H. Lee, "Network-based fire-detection system via controller area network for smart home automation," IEEE Trans., Consumer Electronics, vol.50, no.4, pp.1093-1100, Nov. 2004

[3]. D. Han and J. Lim. "Smart home energy management system using IEEE 802.15. 4 and zigbee," IEEE Trans., Consumer Electronics, vol.56, no.3, pp,1403-1410, Aug. 2010

\section{Bibliography}

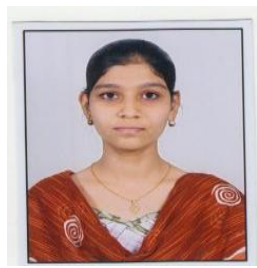

\section{Author 1: Mubeena Begum}

Pursuing M.Tech (VLSI design \& embedded systems) student of BITM College, BELLARY, Karnataka and Completed BE (IT) in PDIT College, HOSPET, Visvesvaraya Technological University, Belgaum, Karnataka, INDIA. With area of interest in embedded system, vlsi design, Network analysis, control system, and VHDL.

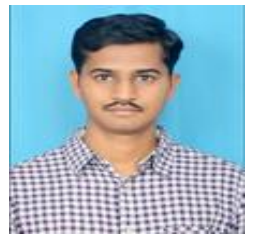

\section{Author 2: Sagara T V}

Obtained M.TECH (DE) in BITM COLEGE,BELLARY,Karnataka,INDIA and obtained $\mathrm{BE}(\mathrm{ECE})$ in Siddaganga institute of technology, Karnataka, INDIA working as Assistant Professor in BITM COLLEGE, BELLARY.Area of interest in communication and internet of things. 


\section{Author 3: Fehmida Begum}

Pursuing BE (ECE) in PDIT College, HOSPET, Visvesvaraya Technological University, Belgaum, Karnataka, INDIA. With area of interest in VHDL, control system, Logic design, and microcontroller. 